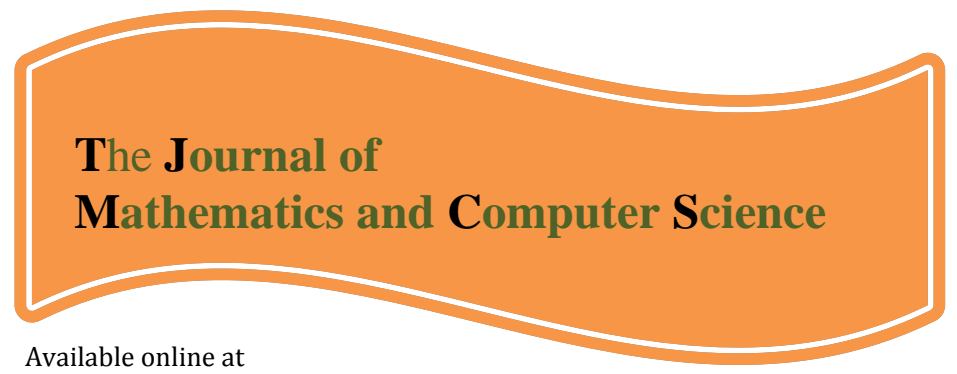

http://www.TJMCS.com

The Journal of Mathematics and Computer Science Vol .1 No.4 (2010) 392-401

\title{
Sequential Sampling Plan by Variable with Fuzzy Parameters
}

\author{
Ezzatallah Baloui Jamkhaneh",1, Bahram Sadeghpour Gildeh ${ }^{2}$ \\ ${ }_{1}^{1}$ Department of Statistics, Islamic Azad University, Gaemshahr Branch, Ghaemshahr, Iran; e_baloui2008@yahoo.com \\ ${ }^{2}$ Department of Statistics, Faculty of Basic Science, University of Mazandaran, Babolsar, Iran; Sadeghpour@umz.ac.ir
}

Received: July 2010, Revised: November 2010

Online Publication: December 2010

\begin{abstract}
In this present paper we have proposed a method for designing sequential sampling plans (SSP) by variable when the acceptable quality levels (AQL) and the rejectable quality levels (RQL) are fuzzy numbers. We are calculated decision criteria in the fuzzy SSP by variable. This plan is well defined since if two quality levels are crisp, it changes to classical plan by variable. For such a plan, a particular table of rejection and acceptance is calculated and compared with the classical one.
\end{abstract}

Keywords: Statistical quality control, sequential sampling plan, fuzzy number, acceptable quality level, lot tolerance percent defective.

\section{Introduction}

Assume that a lot of a great many products is presented for acceptance or rejection inspection. If during the inspection the produced units are only classified in to one of the two disjoint categories: good or bad, when we deal with acceptance sampling by attributes. However, quality specifications are frequently written in terms of measurements like weight, length, diameter, life time, pressure strength, etc. If such continuous quality characteristic is used in acceptance sampling procedure we deal with acceptance sampling by variables. [14]

\footnotetext{
*Corresponding author
} 
Variable sampling plans can also be used to give assurance regarding the average quality of a material, instead of the fraction defective. Sampling plans such as this are most likely to be employed in the sampling of bulk materials that come in bags, drums, or other containers. However, they can also be applied to discrete parts and to other variables, such as energy loss in power transformers. The general approach employed in this type of variables sampling is statistical hypothesis testing. [17]

In sequential sampling, we take a sequence of samples from the lot and allow the number of samples to be determined by the results of the sampling process. To adopt a sequential sampling plan, we should measure the quality of the item in the random sample. Afterwards, by making a comparison between the observations and the limit lines, we can make a decision whether the lot should be accepted or rejected, or a decision should be postponed until a new sample is taken and inspected. If the sample size inspected at each is greater than one, the plan is called group sequential sampling. If the sample size inspected at each stage is one, the plan is called item by item sequential sampling. To design a sampling plan, we need to determine the sample size and limit lines.

Let $\mathrm{X}$ denotes quality characteristics under study production items. The usual assumption is the quality characteristic $(\mathrm{X})$ is normally distribution and that standard deviation of the lot or process is known. The item by item sequential sampling plan by variables plots the cumulative average of the measurements on the quality characteristic. Duncan in [11] provides a good discussion of the design of these plans. [17]

Sequential sampling was developed 1943 for use in rapid quality inspection of war research and production. SSP have been studied by many researchers. They are thoroughly elaborated by Hardeo Sahai et al.. Item by item sequential sampling is based on the sequential probability ratio test (SPRT) developed by Wald [24]. Decision making in Wald SPRT is based on the exact hypotheses, but this precision is not true in real word and there also exist some uncertainty in the value of parameter obtained from experiment or estimation. In the design sequential plan two levels quality AQL and lot tolerance percent defective (LTPD) are crisp. Alternate name for the RQL is LTPD). As there are many different situations in which the AQL and LTPD are imprecise. In fact with this problem we are defining the imprecise parameters as a fuzzy number and we will use from fuzzy SPRT. Testing fuzzy hypotheses was discussed by Arnold [1],[2], Delgado et al. [12], Watanabe and Imaizumi [25], Taheri and Behboodian [20], Torabi and Behboodian [21], Torabi and et al. [22], Baloui Jamkhaneh and Nadi Ghara [3],[4]. SPRT for the fuzzy hypotheses were discussed by Torabi et al. [23]. Chakraborty [9],[10] addresses the problem of designing single stage Dodge Romig lot tolerance percent defective (LTPD) sampling plans when the LTPD, consumer's risk and incoming quality level are modeled using triangular fuzzy numbers. Grzegrozewski [14],[15] also considered sampling plan by variables with fuzzy requirements. Accepted sampling plan with fuzzy parameter were considered by Sadeghpour et al. [19] and Baloui Jamkhaneh et al. [5]-[8].

The paper is organized as follows. The next section introduces SPRT for fuzzy hypotheses testing. In the third section we provide the sequential sampling plan with fuzzy parameter, was considered broadly, and its limit lines in special case was computed. The results are summarized in the concluding section.

\section{SPRT for fuzzy hypotheses testing}

In this section, we define the sequential probability ratio test for fuzzy hypotheses testing (FHT). In FHT with crisp data, fuzzy hypotheses are

$$
\left\{\begin{array}{l}
H_{0}: \theta \text { is } H_{0}(\theta) \\
H_{1}: \theta \text { is } H_{1}(\theta)
\end{array}\right.
$$

Where $H_{j}: \theta$ is $H_{j}(\theta) ; j=0,1$ implied that $\theta$ is in a fuzzy set of $\Theta$ (the parameter space) with membership function $H_{j}(\theta)$ i.e. a function from $\Theta$ to $[0,1]$. Note that the crisp hypotheses 
$H_{j}: \theta$ is $H_{j}(\theta) ; j=0,1$ is a fuzzy hypotheses with membership function $H_{j}(\theta)=1$ at $\theta \in \Theta_{j}$, and zero otherwise.

Let random variable $\mathrm{X}$ have the probability density function (PDF) $f(x, \theta)$. Under the hypotheses $H_{j}(\theta) ; j=0,1$ the weighted probability density function (WPDF) of X is as follows

$$
f_{j}(x)=\int_{\Theta} H_{j}^{*}(\theta) f(x, \theta) d \theta ; j=0,1
$$

That $H_{j}^{*}(\theta)$ is called the pseudo-membership function $H_{j}(\theta)$ and defined by

$$
H_{j}^{*}(\theta)=\frac{H_{j}(\theta)}{\int_{\Theta} H_{j}(\theta) d \theta} ; j=0,1
$$

Substitute $\int$ is $\sum$ in the case that has just countable values. Note that $f_{j}(x)$ is a PDF.

If $X=\left(X_{1}, X_{2}, \ldots, X_{n}\right)$ is a random sample a parametric population with the PDF $f(x, \theta)$, then the joint WPDF of $X$ is

$$
f_{j}(x)=\Pi_{i=1}^{n} f_{j}\left(x_{i}\right) ; j=0,1
$$

and $\Phi(X)$ is a test function, it is the probability of rejecting $\mathrm{H}_{0}$ provided that $X=x$ is observed. The probability of type I and II error of $\Phi(X)$ for the fuzzy testing problem (1) is $\alpha_{\Phi}=E_{0}(\Phi(X))$ and $\beta_{\Phi}=1-E_{1}(\Phi(X))$, respectively, in which $E_{j}(\Phi(X))$ mean the expected value of $\Phi(X)$ over the joint WPDF $f_{j}(x), j=0,1$.

Let $X_{1}, X_{2}, \ldots$ denote a sequence of the iid random variables, from a population with PDF $f(x, \theta)$. First compute sequentially $R_{1}, R_{2}, \ldots$. That

$$
\begin{aligned}
R_{m}\left(x_{1}, x_{2}, \ldots, x_{m}\right) & =\frac{L_{0}\left(x_{1}, x_{2}, \ldots, x_{m}\right)}{L_{1}\left(x_{1}, x_{2}, \ldots, x_{m}\right)} \\
& =\prod_{i=1}^{m} \frac{f_{0}\left(x_{i}\right)}{f_{1}\left(x_{i}\right)} \\
& =\prod_{i=1}^{m} \frac{\int_{\Theta} H_{0}^{*}(\theta) f\left(x_{i} ; \theta\right) d \theta}{\int_{\Theta} H_{1}^{*}(\theta) f\left(x_{i} ; \theta\right) d \theta} .
\end{aligned}
$$

For fixed $k_{0}$ and $k_{1}$ satisfying $0<k_{0}<k_{1}$, adopt the following procedure: take observation $x_{1}$ and compute $R_{1}$; if $R_{1} \leq k_{0}$, reject $H_{0}$; if $R_{1} \geq k_{1}$, accept $H_{0}$; and if $k_{0}<R_{1}<k_{1}$, take observation $x_{2}$, and compute $R_{2}$; if $R_{2} \leq k_{0}$, reject $H_{0}$; if $R_{2} \geq k_{1}$, accept $\mathrm{H}_{0}$; and if $k_{0}<R_{2}<k_{1}$, take observation $x_{3}$, etc. The idea is to continue sampling as long as $k_{0}<R_{m}<k_{1}$ and stop as soon as $R_{m} \leq k_{0}$ or $R_{m} \geq k_{1}$, rejecting $H_{0}$ if $R_{m} \leq k_{0}$ and accepting $\mathrm{H}_{0}$ if $R_{m} \geq k_{1}$. Consequently, the critical region of the described SPRT for fuzzy hypotheses testing (1) is $C=\cup_{n=1}^{\infty} C_{n}$, where

$$
C_{n}=\left\{\left(x_{1}, \ldots, x_{n}\right) \mid k_{0}<R_{j}\left(x_{1}, \ldots, x_{j}\right)<k_{1}, j=1, \ldots, n-1, R_{n}\left(x_{1}, \ldots, x_{n}\right) \leq k_{0}\right\}
$$


Similarly, the acceptance region can be as $A=\cup_{n=1}^{\infty} A_{n}$, where

$$
A_{n}=\left\{\left(x_{1}, \ldots, x_{n}\right) \mid k_{0}<R_{j}\left(x_{1}, \ldots, x_{j}\right)<k_{1}, j=1, \ldots, n-1, R_{n}\left(x_{1}, \ldots, x_{n}\right) \geq k_{1}\right\} .
$$

Therefore in the SPRT for fuzzy hypotheses, the probability of type I and II errors is calculated by

$$
\alpha_{\Phi}=\sum_{n=1}^{\infty} \int_{C_{n}} L_{0}(X) d X \quad, \quad \beta_{\Phi}=\sum_{n=1}^{\infty} \int_{A_{n}} L_{1}(X) d X .
$$

Let $k_{0}$ and $k_{1}$ be defined so that the SPRT for fuzzy hypotheses has the fixed probability of type I and II errors $\alpha$ and $\beta$. Then $k_{0}$ and $k_{1}$ can be approximated by $k_{0}^{\prime}=\frac{\alpha}{1-\beta}$ and $k_{1}^{\prime}=\frac{1-\alpha}{\beta}$. If $\alpha^{\prime}$ and $\beta^{\prime}$ are the error size of the SPRT defined by $k_{0}^{\prime}$ and $k_{1}^{\prime}$, then $\alpha^{\prime}+\beta^{\prime} \leq \alpha+\beta$. If

$$
Z_{i}=\operatorname{Ln} \frac{\int_{\Theta} H_{0}^{*}(\theta) f\left(x_{i} ; \theta\right) d \theta}{\int_{\Theta} H_{1}^{*}(\theta) f\left(x_{i} ; \theta\right) d \theta} .
$$

With observed one sample and using (3), an equivalent test to the SPRT for fuzzy hypotheses is given by the following: continue sampling as long as $\operatorname{Ln}\left(k_{0}^{\prime}\right)<\sum_{i=1}^{m} Z_{i}<\operatorname{Ln}\left(k_{1}^{\prime}\right)$, otherwise stop sampling, if $\sum_{i=1}^{m} Z_{i} \leq \operatorname{Ln}\left(k_{0}^{\prime}\right)$ then we will reject $H_{0}$ and if $\sum_{i=1}^{m} Z_{i} \geq \operatorname{Ln}\left(k_{1}^{\prime}\right)$ then we will accept $H_{0}$.

\section{SSP with fuzzy parameters}

Decision criteria in an item by item sequential sampling plan are the acceptance and rejection lines. This two limit lines are plotted in terms of the cumulative average of the measurements on the quality characteristic selected up to that time. The operation of such that plan is illustrated in Figure 1. For each point in this figure, the x-axis is the total number of items selected up to that time, and the $y$-axis is the cumulative average of the measurements on the quality characteristic. Then operation procedure is given in the following:

(i) If plotted points stay within the boundaries of the limit lines, another sample must be drown,

(ii) When plotted point falls on or above the upper line, in the stage the lot is rejected,

(iii) When plotted point falls on or below the lower line, in the stage the lot is accepted. 


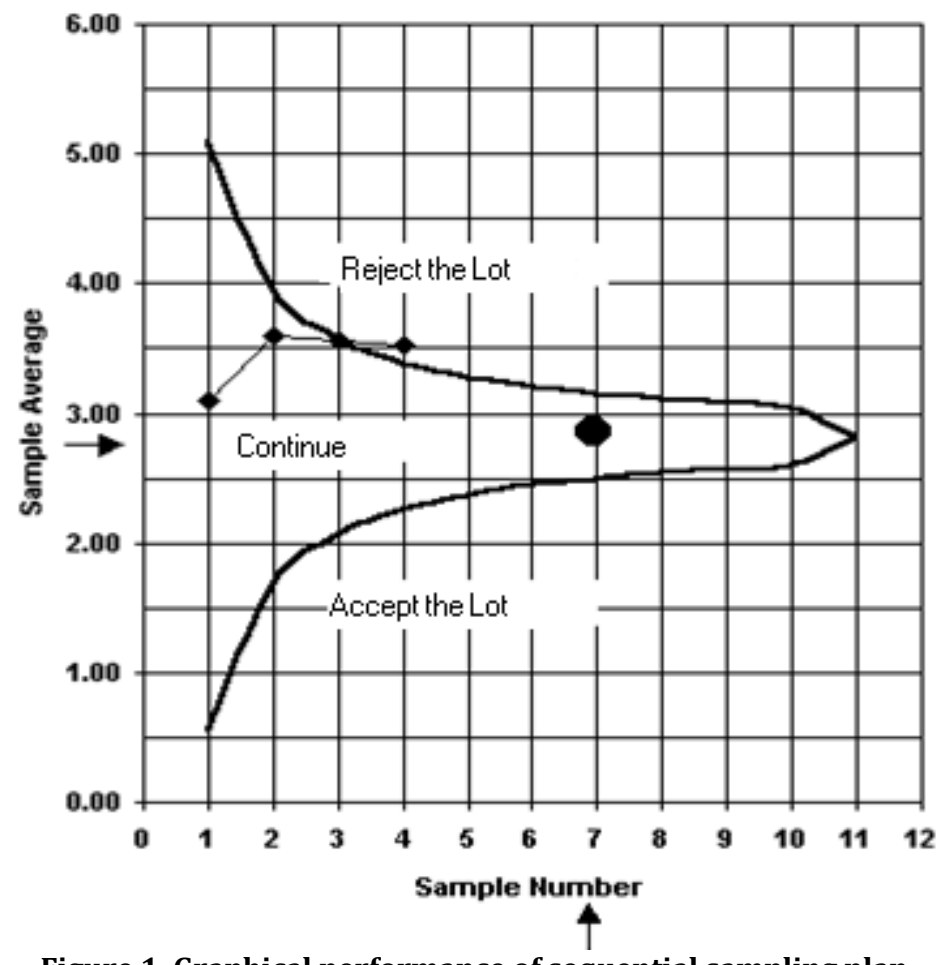

Figure 1. Graphical performance of sequential sampling plan

Accepting or rejecting a lot in the SSP analogous not rejecting or rejecting the null hypotheses in a hypotheses test. The hypotheses for SSP as a kind of statistical test are:

$\mathrm{H}_{0}$ : The lot is of acceptable quality level (AQL)

$\mathrm{H}_{1}$ : The lot is of reject able quality level (RQL)

The AQL presents the poorest level of quality for the vender's process that the consumer would consider to be acceptable as a process average. The RQL is the forest level of quality that the consumer is willing to accept in an individual lot. Probability of type I and II errors $\alpha$ and $\beta$ for this hypotheses test is as follows

$$
\alpha=P\left(R H_{0} \mid H_{0}\right), \beta=P\left(A H_{0} \mid H_{1}\right) .
$$

A lot may be rejected that should be accepted and the risk of doing this is the producer's risk $(\alpha)$. The second error is that a lot may be accepted that should be rejected and the risk of doing is called the consumer's risk $(\beta)$. If $A Q L=\mu_{1}$ and $R Q L=\mu_{2}\left(\mu_{1}<\mu_{2}\right)$ then an equivalent hypothesis is given by the following:

$$
\left\{\begin{array}{l}
H_{0}: \mu=\mu_{1}, \\
H_{1}: \mu=\mu_{2} .
\end{array}\right.
$$

When designing an item by item sequential sampling plan, four parameters of the AQL, the producer's risk $(\alpha)$ (the probability of rejecting a lot of AQL quality), RQL and the consumer's risk $(\beta)$ (the probability of accepting a lot of RQL quality ) must be determined prior to determining the acceptation and rejection lines. In the design SSP two levels of quality are crisp. But sometimes these are not exact and certain. So, we fact a fuzzy hypotheses as follows 


$$
\left\{\begin{array}{l}
H_{0}: \mu \approx \mu_{1}, \\
H_{1}: \mu \approx \mu_{2} .
\end{array}\right.
$$

We propose to design SSP by variables with fuzzy AQL and fuzzy RQL. Such this plan is based on the SPRT for fuzzy hypotheses. In FHT with crisp data, fuzzy hypotheses are

$$
\left\{\begin{array}{l}
H_{0}: \mu \text { is } H_{0}(\mu) \\
H_{1}: \mu \text { is } H_{1}(\mu)
\end{array}\right.
$$

Where membership function of $H_{j}(\mu)$ is

$$
H_{j}(\mu)=e^{\frac{-\left(\mu-\mu_{j}\right)^{2}}{2 \sigma_{0}^{2}}}, j=0,1
$$

And pseudo membership function of $H_{j}(\mu)$ is

$$
H_{j}^{*}(\mu)=\frac{1}{\sqrt{2 \pi} \sigma_{0}} e^{\frac{-\left(\mu-\mu_{j}\right)^{2}}{2 \sigma_{0}^{2}}}, j=0,1
$$

it is easy to show that

$$
f_{j}(x)=\frac{1}{\sqrt{2 \pi\left(\sigma^{2}+\sigma_{0}^{2}\right)}} e^{\frac{-\left(x-\mu_{j}\right)^{2}}{2\left(\sigma^{2}+\sigma_{0}^{2}\right)}}, j=0,1
$$

Finally we obtain

$$
\begin{aligned}
R_{n}\left(x_{1}, x_{2}, \ldots, x_{n}\right)= & \frac{\Pi_{i=1}^{n} \frac{1}{\sqrt{2 \pi\left(\sigma^{2}+\sigma_{0}^{2}\right)}} \exp \left\{\frac{-\left(x_{i}-\mu_{1}\right)^{2}}{2\left(\sigma^{2}+\sigma_{0}^{2}\right)}\right\}}{\prod_{i=1}^{n} \frac{1}{\sqrt{2 \pi\left(\sigma^{2}+\sigma_{0}^{2}\right)}} \exp \left\{\frac{-\left(x_{i}-\mu_{2}\right)^{2}}{2\left(\sigma^{2}+\sigma_{0}^{2}\right)}\right\}} \\
& =\prod_{i=1}^{n} \exp \left\{x_{i}\left(\frac{\mu_{1}-\mu_{2}}{\sigma^{2}+\sigma_{0}^{2}}\right)-\frac{\mu_{1}^{2}-\mu_{2}^{2}}{2\left(\sigma^{2}+\sigma_{0}^{2}\right)}\right\}
\end{aligned}
$$

if

$$
Z_{i}=X_{i}\left(\frac{\mu_{1}-\mu_{2}}{\sigma^{2}+\sigma_{0}^{2}}\right)-\frac{\mu_{1}^{2}-\mu_{2}^{2}}{2\left(\sigma^{2}+\sigma_{0}^{2}\right)} .
$$


Then at of sampling, lot the $\mathrm{n}^{\text {th }}$ stage reject the

\begin{tabular}{|c|c|c|c|c|c|}
\hline$n$ & Ac. Number & Re. Number & $n$ & Ac. Number & Re. Number \\
\hline 1 & 3.6 & 5.66 & 7 & 4.37 & 4.67 \\
\hline 2 & 4.01 & 5.08 & 8 & 4.39 & 4.64 \\
\hline 3 & 4.2 & 4.88 & 9 & 4.4 & 4.63 \\
\hline
\end{tabular}

if $\sum_{i=}^{n} Z_{i} \leq \operatorname{Ln} \frac{\alpha}{1-\beta}$, accept the lot if $\sum_{i=}^{n} Z_{i} \geq \operatorname{Ln} \frac{1-\alpha}{\beta}$ and continue sampling by taking an additional observation if $\operatorname{Ln} \frac{\alpha}{1-\beta}<\sum_{i=}^{n} Z_{i}<\operatorname{Ln} \frac{1-\alpha}{\beta}$. Using the parameters $\mu_{1}, \mu_{2}, \alpha$ and $\beta$ the SSP with fuzzy parameter is determined by the acceptance and rejection lines given as follows

$$
\bar{X}_{A}=s+\frac{h_{1}}{n} \quad \text { (acceptance line) }, \quad \bar{X}_{R}=s+\frac{h_{2}}{n} \quad \text { (rejection line) }
$$

Here

$$
\begin{gathered}
k=\frac{\mu_{1}-\mu_{2}}{\sigma^{2}+\sigma_{0}^{2}} \quad, \quad s=\frac{\frac{\mu_{1}^{2}-\mu_{2}^{2}}{2\left(\sigma^{2}+\sigma_{0}^{2}\right)}}{k} \\
h_{1}=\frac{\operatorname{Ln} \frac{1-\alpha}{\beta}}{k} \quad, \quad h_{2}=\frac{\operatorname{Ln} \frac{\alpha}{1-\beta}}{k} .
\end{gathered}
$$

For example, suppose we will be to find a SSP for which $\mu_{1}$ is close to $4, \alpha=0.05, \mu_{2}$ is close to 5 and $\beta=0.1, \sigma^{2}=0.3$ and $\sigma_{0}^{2}=0.1$ thus, $k=-2.5, s=4.5, h_{1}=-0.9005, h_{2}=1.156$ therefore, the acceptance and rejection lines are

$$
\bar{X}_{A}=4.5-\frac{0.9005}{n} \quad, \quad \bar{X}_{R}=4.5+\frac{1.1561}{n} \text {. }
$$

Table 1: Fuzzy sequential sampling plan $\sigma^{2}=0.3, \sigma_{0}^{2}=0.1, \mu_{1} \approx 4, \alpha=0.05, \mu_{2} \approx 5, \beta=0.1$ 
Ezzatallah Baloui Jamkhaneh, Bahram Sadeghpour Gildeh/ TJMCS Vol .1 No.4 (2010) 392-401

\begin{tabular}{|c|c|c|c|c|c|}
\hline 4 & 4.27 & 4.79 & 10 & 4.41 & 4.62 \\
\hline 5 & 4.32 & 4.73 & 11 & 4.42 & 4.61 \\
\hline 6 & 4.31 & 4.69 & 12 & 4.42 & 4.6 \\
\hline
\end{tabular}

The values of acceptance and rejection lines are shown in Table 1 . For example, if $n=10$ then

(i) Accept lot, if cumulative average of the measurements on the quality characteristic is less than or equal to 4.41 .

(ii) Continue sampling, if cumulative average of the measurements on the quality characteristic is in $(4.41,4.62)$.

(iii) Reject lot, if cumulative average of the measurements on the quality characteristic is greater than or equal to 4.62 .

Table 2: Decision making base on fuzzy SSP

\begin{tabular}{|c|c|c|c|c|c|c|c|}
\hline \multicolumn{5}{|c|}{ First lot } & \multicolumn{5}{c|}{ Second lot } \\
\hline$n$ & $x_{i}$ & $\bar{x}$ & decision & $n$ & $x_{i}$ & $\bar{x}$ & decision \\
\hline 1 & 4.1 & 4.1 & continue & 1 & 4.2 & 4.2 & continue \\
\hline 2 & 4.5 & 4.3 & continue & 2 & 4.5 & 4.35 & continue \\
\hline 3 & 4.2 & 4.27 & continue & 3 & 5.1 & 4.6 & continue \\
\hline 4 & 5 & 4.45 & continue & 4 & 5.4 & 4.8 & reject \\
\hline 5 & 3.7 & 4.3 & accept & & & & \\
\hline
\end{tabular}

Table 3: Traditional sequential sampling plan $\sigma^{2}=0.3, \sigma_{0}^{2}=0, \mu_{1} \approx 4, \alpha=0.05, \mu_{2} \approx 5, \beta=0.1$

Table 1

\begin{tabular}{|c|c|c|c|c|c|}
\hline$n$ & Ac. Number & Re. Number & $n$ & Ac. Number & Re. Number \\
\hline 1 & 3.82 & 5.37 & 7 & 4.4 & 4.62 \\
\hline 2 & 4.16 & 5.93 & 8 & 4.42 & 4.61 \\
\hline 3 & 4.27 & 4.8 & 9 & 4.42 & 4.6 \\
\hline 4 & 4.33 & 4.72 & 10 & 4.43 & 4.59 \\
\hline 5 & 4.36 & 4.67 & 11 & 4.44 & 4.58 \\
\hline 6 & 4.39 & 4.64 & 12 & 4.44 & 4.57 \\
\hline
\end{tabular}

shows that with the increasing of $n$, the width of continue sampling band will be narrower. The ambiguity amount of $\mu_{j}$ in the amount of $\sigma_{0}^{2}$ is hidden. With the reduction of $\sigma_{0}^{2}$, the ambiguity amount in $\mu_{j}$ will be less and if the $\sigma_{0}^{2}$ be zero, $H_{j}(\mu)$ will be one for $\mu_{j}$ and will be zero in other points. This means that $\mu_{j}$ will change to crisp state. With increasing or decreasing of ambiguity amount in $\mu_{j}$, the width of continue sampling band will be wide and narrow respectively. With the decreasing of $\mu_{j}$ ambiguity,the rejection and acception lines in fuzzy state will closed to crisp state, and when the $\sigma_{0}^{2}$ become to zero, the fuzzy state change to crisp state. Table 2 shows decision making about two lots. 


\section{Conclusion}

In this paper we have proposed a method to design SSP with fuzzy AQL and fuzzy LTPD. This plan is well defined since, if the parameters are crisp, it changes to classical plan. Comparing Table 1 and the table related to SSP in its classical state in terms of common parameters (Table 3), one can conclude that this plan acceptance and rejection of lots happens cautiously, this means that fuzzy method is decided with more review. However, changing $\sigma_{0}^{2}$ and correspondingly, changing the skewed of $H_{j}(\mu)$, the difference between the acceptance and rejection numbers of these two plans would change. In such a plan with increasing sample size, the width of continues sampling band will be narrower.

\section{References}

[1] Arnold, B.F., "Statistical tests optimally meeting certain fuzzy requirements on the power function and on the sample size", Fuzzy Sets and System, 75(2), pp. 365-372, 1995

[2] Arnold, B.F., "An approach to fuzzy hypotheses testing", Metrika, 44, pp. 119-126, 1996.

[3] Baloui Jamkhaneh, E., and Nadi Ghara, A., "Testing Statistical Hypotheses for Compare Means with Vague Data", International Mathematical Forum, 5(13), pp. 615 - 620, 2010.

[4] Baloui Jamkhaneh E., and Nadi Ghara, A., "Testing Statistical Hypotheses with Fuzzy Data", Proceedings of International Conference on Intelligent Computing and Cognitive Informatics, pp. 86-89, 2010.

[5] Baloui Jamkhaneh, E., Sadeghpour Gildeh, B., and Yari, Gh., "Acceptance single sampling plan with fuzzy parameter", Iranian Journal of Fuzzy Systems, (to appear).

[6] Baloui Jamkhaneh, E., Sadeghpour Gildeh, B., and Yari, Gh., "Inspection Error and its Effects on Single Sampling Plans with Fuzzy Parameters", Structural and Multidisciplinary Optimization, (to appear).

[7] Baloui Jamkhaneh, E., Sadeghpour Gildeh, B., "AOQ and ATI for Double Sampling Plan with Using Fuzzy Binomial Distribution", Proceedings of International Conference on Intelligent Computing and Cognitive Informatics, pp. 45-49, 2010.

[8] Baloui Jamkhaneh, E., Sadeghpour Gildeh, B. and Yari, Gh., "Important Criteria of Rectifying Inspection for

Single Sampling Plan with Fuzzy Parameter", Int. J. Contemp. Math. Sciences, 4, (3)6, pp. 1791 - 1801, 2009.

[9 ] Chakraborty, T.K., "A class of single sampling plans based on fuzzy optimization", Opsearch, 29(1), pp. 11-20,1992.

[10 ] Chakraborty, T.K., "Possibilistic parameter single sampling inspection plans", Opsearch, 31(2), pp. 108-126, 1994.

[11] Dancan, A. J., "Quality control and industrial statistics", 5th ed. Irwin, Homewood, IL., 1986.

[12] Delgado M., Verdegay M. A. and Vila M. A., "Testing fuzzy hypotheses: a Bayesian approach", in: M.M. Gupta et al.(Eds), Approximate Reasoning in Expert Systems, North-Holland Publishing Co, Amsterdam, pp. 307-316, 1985.

[13] Dumicic, K., Bahovec, V., Zivadinovic, N. K., "Studying an OC curve of an acceptance sampling: A statistical quality control tool", proceeding of the 7th WSEAS international conference on mathematics \& computers in business \& economics, cavtat, croatia, JUNE 13-15, pp. 1-6, 2006.

[14] Grzeqorzewski, P.,"A soft design of acceptance sampling plan by variables", in: Technologies for constructing intelligent systems, Eds, Springer, 2, pp. 275-286, 2002.

[15] Grzegorzewski, P., " Acceptance sampling plans by attributes with fuzzy risks and quality levels", in: Frontiers in frontiers in statistical quality control. Vol. 6, Eds. Wilrich P. Th. Lenz H. J. Springer, Heidelberg, pp. 36-46, 2001. 
[16] Hardeo, S., Anwer, K., and Muhammad, A., "A visual basic program to determine Wald's sequential sampling plan", 24(3), 2003.

[17] Montgomery, D. C., "introduction to statistical quality control", Wiley New York, 1991.

[18] Montgomry , D. C., and Runger G. C., "Applied statistics and probability for engineers", 2nd ed., Wiley, New York, 1999.

[19] Sadeghpour Gildeh, B., Yari, Gh., and Baloui Jamkhaneh, E., "Acceptance double sampling plan with fuzzy parameter", proceedings of the 11th joint conference on information science, Shenzhen, China,2008.

[20] Tahiri, S.M.,and Behboodian, J., "A Bayesian approach to fuzzy hypotheses testing", Fuzzy Sets and Systems, 123, pp.39-48, 2001.

[21] Torabi H., and Behboodian J., "Likelihood ratio test for fuzzy hypotheses testing", Statistical Papers, 48(3), pp. 509-522, 2007.

[22 ] Torabi, H., Behboodian, J., and Taheri. S.M., "Neyman-Pearson lemma for fuzzy hypotheses testing with vague data", Metrika, 64(3), pp. 289-304, 2006.

[23] Torabi, H., and Mirhosseini S. M.,"Sequential probability ratio tests for fuzzy hypotheses testing", Applied mathematical sciences, 3(33), pp. 1609-1618, 2009.

[24] Wald, A., "Sequential analysis", John Wiley, New York, 1947.

[25] Watanabe, N., Imaizumi, T.,"A fuzzy statistical test of fuzzy hypotheses", Fuzzy Sets and Systems, 53, pp.167-178, 1993. 